\title{
A GENERALIZED BETA FUNCTION AND ASSOCIATED PROBABILITY DENSITY
}

\author{
Y. BEN NAKHI and S. L. KALLA
}

Received 30 April 2001

\begin{abstract}
We introduce and establish some properties of a generalized form of the beta function. Corresponding generalized incomplete beta functions are also defined. Moreover, we define a new probability density function (pdf) involving this new generalized beta function. Some basic functions associated with the pdf, such as moment generating function, mean residue function, and hazard rate function are derived. Some special cases are mentioned. Some figures for pdf, hazard rate function, and mean residue life function are given. These figures reflect the role of shape and scale parameters.
\end{abstract}

2000 Mathematics Subject Classification: 33C20, 60E10, $62 \mathrm{E} 15$.

1. Introduction. Recently, many authors have defined and studied generalized form of different special functions [4, 5, 8, 12, 13]. Virchenko et al. [12] have treated a generalized gamma function in the form,

$$
\stackrel{\omega}{D}\left(\begin{array}{c}
a, b ; c ; v \\
u, \mu
\end{array}\right)=v^{-a} \int_{0}^{\infty} t^{u-1} e^{-p t}{ }_{2} R_{1}\left(a, b ; c ;-\frac{t}{v}\right) d t
$$

where $a, b$, and $c$ are complex parameters, $\omega>0, \operatorname{Re} p, \operatorname{Re} u>0,|\arg v|<\pi$, and $c \neq 0,-1,-2, \ldots$. The case where $\omega=p=1, b=c$ reduces to Kobayashi's generalized gamma function [8]. Moreover, if we take $a=0$ in this equation, we get the well-known gamma function. Kalla and Al-Saqabi [6] have used this function to define a probability density function, which generalizes results of Kobayashi [8] and Kalla et al. [7].

DEFINITION 1.1. Continue with the preceding assumptions on the parameters $a$, $b, c, \omega, u$, and $v$. Then for $\operatorname{Re}(a+\mu)$ and $\operatorname{Re}(b+\mu)>0$, we define a generalized form of the beta function as

$$
\stackrel{\omega}{B}\left(\begin{array}{c}
a, b ; c ; v \\
u, \mu
\end{array}\right) \triangleq v^{-a} \int_{0}^{\infty} t^{u-1}(1+t)^{-\mu-u}{ }_{2} \stackrel{\omega}{R}_{1}\left(a, b ; c ;-\frac{t}{v}\right) d t
$$

where ${ }_{2} \stackrel{\omega}{R}_{1}(a, b ; c ; x)$ is the $\omega$-Gauss hypergeometric function $[12,13]$ whose series representation is given by,

$$
{ }_{2} R_{1}^{\omega}(a, b ; c ; x)=\frac{\Gamma(c)}{\Gamma(b)} \sum_{k=0}^{\infty} \frac{(a)_{k} \Gamma(b+\omega k)}{\Gamma(c+\omega k)} \frac{x^{k}}{k !}, \quad|x|<1
$$


and its integral representation in the form,

$$
\begin{aligned}
{ }_{2}^{\omega} R_{1}(a, b ; c ; x) & =L \int_{0}^{1} t^{b-1}(1-t)^{c-b-1}\left(1-x t^{\omega}\right)^{-a} d t \\
& =\frac{L}{\omega} \int_{0}^{1} t^{b / \omega-1}\left(1-t^{1 / \omega}\right)^{c-b-1}(1-x t)^{-a} d t,
\end{aligned}
$$

where

$$
\operatorname{Re}(c)>\operatorname{Re}(b)>0, \quad L=\frac{\Gamma(c)}{\Gamma(b) \Gamma(c-b)} .
$$

For $\omega=1,(1.4)$ is the classical Gauss hypergeometric function, hence (1.2) becomes

$$
B\left(\begin{array}{c}
a, b ; c ; v \\
u, \mu
\end{array}\right)=v^{-a} \int_{0}^{\infty} t^{u-1}(1+t)^{-\mu-u}{ }_{2} F_{1}\left(a, b ; c ;-\frac{t}{v}\right) d t,
$$

and by letting $b=c$, we have [11]

$$
\begin{aligned}
B\left(\begin{array}{c}
a, b ; b ; v \\
u, \mu
\end{array}\right) & =\int_{0}^{\infty} t^{u-1}(1+t)^{-\mu-u}(v+t)^{-a} d t \\
& =v^{-a} B(u, \mu+a)_{2} F_{1}\left(u, a ; u+a+\mu ; 1-\frac{1}{v}\right) .
\end{aligned}
$$

Further, if we take $a=0$ then (1.2) reduces to the well-known beta function, that is,

$$
B(u, \mu)=\int_{0}^{\infty} t^{u-1}(1+t)^{-\mu-u} d t, \quad \operatorname{Re}(u), \operatorname{Re}(\mu)>0 .
$$

In Section 2, we establish a number of analytic properties, such as recurrence relations and the asymptotic expansions for our generalized beta function. We express this generalized beta function in terms of $\omega$-hypergeometric functions [13]

$$
{ }_{3} R_{2}\left(\begin{array}{c}
a_{1}, a_{2}: b ; x \\
c: d
\end{array}\right) \triangleq \frac{\Gamma(c)}{\Gamma(b)} \sum_{k=0}^{\infty} \frac{\left(a_{1}\right)_{k}\left(a_{2}\right)_{k} \Gamma(b+\omega k)}{(c)_{k} \Gamma(d+\omega k)} \frac{x^{k}}{k !} .
$$

In Section 3, we define generalized incomplete beta functions associated with the function defined by (1.2). These incomplete forms of the generalized beta functions are used to study some statistical functions in later sections. Moreover, we introduce and study a new probability density function (pdf) involving the generalized beta function in Section 4. Finally, we derive some basic functions associated with this density function, namely, the $k$ th moment, moment generating function, the hazard rate function, and the mean residue life function. Corresponding results for beta distribution are listed. Some figures are given for pdf, hazard rate function, and mean residue life function.

2. The generalized beta function. We begin this section by observing that (1.2) can be rewritten as

$$
\stackrel{\omega}{B}\left(\begin{array}{c}
a, b ; c ; v \\
u, \mu
\end{array}\right)=v^{u-a} \int_{0}^{\infty} t^{u-1}(1+v t)^{-\mu-u}{ }_{2} \stackrel{\omega}{R}_{1}(a, b ; c ;-t) d t .
$$


We express this generalized beta function in terms of $\omega$-hypergeometric functions in the following theorem.

THEOREM 2.1. The generalized beta function $\stackrel{\omega}{B}(\underset{a, b ; c ; v}{u, \mu})$ can be represented as

$$
\stackrel{\omega}{B}\left(\begin{array}{c}
a, b ; c ; v \\
u, \mu
\end{array}\right)=\stackrel{\omega}{E}_{1}\left(\begin{array}{c}
a, b ; c ; v \\
u, \mu
\end{array}\right)+\stackrel{\omega}{E}_{2}\left(\begin{array}{c}
a, b ; c ; v \\
u, \mu
\end{array}\right)
$$

where,

$$
\begin{aligned}
& \stackrel{\omega}{E}_{1}\left(\begin{array}{c}
a, b ; c ; v \\
u, \mu
\end{array}\right)=v^{-a} \times B(u, \mu) \times{ }_{3} R_{2}\left(\begin{array}{c}
u, a: b ; \frac{1}{v} \\
1-\mu: c
\end{array}\right) \\
& \stackrel{\omega}{E}\left(\begin{array}{c}
a, b ; c ; v \\
u, \mu
\end{array}\right)=v^{-a-\mu} \times \stackrel{\omega}{\Gamma}(*) \times{ }_{3} R_{2}\left(\begin{array}{c}
a+\mu, u+\mu: b+\omega \mu ; \frac{1}{v} \\
1+\mu: c+\omega \mu
\end{array}\right)
\end{aligned}
$$

with

$$
\stackrel{\omega}{\Gamma}(*)=\Gamma\left(\begin{array}{c}
c, a+\mu, b+\omega \mu,-\mu \\
a, b, c+\omega \mu
\end{array}\right) .
$$

Proof. Using (1.4), the integral representation for ${ }_{2} R_{1}$, yields

$$
\begin{aligned}
\stackrel{\omega}{B}\left(\begin{array}{c}
a, b ; c ; v \\
u, \mu
\end{array}\right) & =v^{-a} \int_{0}^{\infty} x^{u-1}(1+x)^{-\mu-u_{2} R_{1}}\left(a, b ; c ;-\frac{x}{v}\right) d x \\
& =v^{-a} L_{1} \int_{0}^{1} t^{b-1}(1-t)^{c-b-1}\left(\int_{0}^{\infty} x^{u-1}(1+x)^{-\mu-u}\left(1+\frac{t^{\omega}}{v} x\right)^{-a} d x\right) d t \\
& =v^{-a} L_{2} \int_{0}^{1} t^{b-1}(1-t)^{c-b-1}{ }_{2} F_{1}\left(a, u ; a+u+\mu ; 1-\frac{t^{\omega}}{v}\right) d t
\end{aligned}
$$

where

$$
L_{1}=\frac{1}{B(b, c-b)}, \quad L_{2}=\frac{B(u, a+\mu)}{B(b, c-b)} .
$$

Using the transformation formulae [1, 9], yields

$$
F=L_{3} \times{ }_{2} F_{1}\left(a, u ; 1-\mu ; \frac{t^{\omega}}{v}\right)+L_{4} \times\left(\frac{t^{\omega}}{v}\right)^{\mu} \times{ }_{2} F_{1}\left(u+\mu, a+\mu ; 1+\mu ; \frac{t^{\omega}}{v}\right),
$$

where

$$
F={ }_{2} F_{1}\left(a, u ; a+u+\mu ; 1-\frac{t^{\omega}}{v}\right), \quad L_{3}=\Gamma\left(\begin{array}{c}
\mu, a+u+\mu \\
a+\mu, u+\mu
\end{array}\right), \quad L_{4}=\Gamma\left(\begin{array}{c}
-\mu, a+u+\mu \\
a, u
\end{array}\right) .
$$


Let

$$
\begin{aligned}
& \stackrel{\omega}{E}_{1}\left(\begin{array}{c}
a, b ; c ; v \\
u, \mu
\end{array}\right) \triangleq v^{-a} \times L_{2} L_{3} \times \int_{0}^{1} t^{b-1}(1-t)^{c-b-1}{ }_{2} F_{1}\left(a, u ; 1-\mu ; \frac{t^{\omega}}{v}\right) d t \\
& =v^{-a} \times L_{2} L_{3} \times \sum_{k=0}^{\infty} \frac{(a)_{k}(u)_{k}}{(1-\mu)_{k}} \times \frac{v^{-k}}{k !} \int_{0}^{1} t^{b+\omega k-1}(1-t)^{c-b-1} d t \\
& =v^{-a} \times B(\mu, u) \times \frac{\Gamma(c)}{\Gamma(b)} \sum_{k=0}^{\infty} \frac{(a)_{k}(u)_{k} \Gamma(b+\omega k)}{v^{k}(1-\mu)_{k} \Gamma(c+\omega k) k !} \\
& =v^{-a} \times B(\mu, u){ }_{3} \stackrel{\omega}{R}_{2}\left(\begin{array}{c}
u, a: b ; \frac{1}{v} \\
1-\mu: c
\end{array}\right), \\
& \stackrel{\omega}{E}_{2}\left(\begin{array}{c}
a, b ; c ; v \\
u, \mu
\end{array}\right) \triangleq \frac{L_{2} L_{4}}{v^{a}} \times\left(\frac{t^{\omega}}{v}\right)^{\mu} \times \int_{0}^{1} t^{b-1}(1-t)^{c-b-1}{ }_{2} F_{1}\left(u+\mu, a+\mu ; 1+\mu ; \frac{t^{\omega}}{v}\right) d t \\
& =\frac{L_{2} L_{4}}{v^{a+\mu}} \sum_{k=0}^{\infty} \frac{(a+\mu)_{k}(u+\mu)_{k}}{(1+\mu)_{k}} \times \frac{v^{-k}}{k !} \int_{0}^{1} t^{b+\omega k+\omega \mu-1}(1-t)^{c-b-1} d t \\
& =v^{-a-\mu} \times \stackrel{\omega}{\Gamma}(*) \times \frac{\Gamma(c+\omega \mu)}{\Gamma(b+\omega \mu)} \sum_{k=0}^{\infty} \frac{(a+\mu)_{k}(u+\mu)_{k} \Gamma(b+\omega \mu+\omega k)}{v^{k}(1+\mu)_{k} \Gamma(c+\omega \mu+\omega k) k !} \\
& =v^{-a-\mu} \times \stackrel{\omega}{\Gamma}(*) \times{ }_{3} \stackrel{\omega}{R}_{2}\left(\begin{array}{c}
a+\mu, u+\mu: b+\omega \mu ; \frac{1}{v} \\
1+\mu: c+\omega \mu
\end{array}\right)
\end{aligned}
$$

and the proof is complete.

REMARK 2.2. For $\omega=1$, we get the representation of $B$ in terms of hypergeometric functions given in [11, page 315], that is,

$$
B\left(\begin{array}{c}
a, b ; c ; v \\
u, \mu
\end{array}\right)=E_{1}\left(\begin{array}{c}
a, b ; c ; v \\
u, \mu
\end{array}\right)+E_{2}\left(\begin{array}{c}
a, b ; c ; v \\
u, \mu
\end{array}\right),
$$

where

$$
\begin{aligned}
& E_{1}\left(\begin{array}{c}
a, b ; c ; v \\
u, \mu
\end{array}\right)=\frac{B(u, \mu)}{v^{a}}{ }_{3} F_{2}\left(\begin{array}{c}
u, a, b ; \frac{1}{v} \\
1-\mu, c
\end{array}\right), \\
& E_{2}\left(\begin{array}{c}
a, b ; c ; v \\
u, \mu
\end{array}\right)=v^{-a-\mu} \times \Gamma(*) \times{ }_{3} F_{2}\left(\begin{array}{c}
u+\mu, a+\mu, b+\mu ; \frac{1}{v} \\
1+\mu, c+\mu
\end{array}\right),
\end{aligned}
$$

with

$$
\Gamma(*)=\Gamma\left(\begin{array}{c}
c, a+\mu, b+\mu,-\mu \\
a, b, c+\mu
\end{array}\right) .
$$

ASYMPTOTIC EXPANSIONS FOR $\stackrel{\omega}{B}\left(\begin{array}{c}a, b ; c ; v \\ u, \mu\end{array}\right)$. Now we establish the asymptotic expansions for the generalized beta function using the previous theorem. 
THEOREM 2.3. Recall ${ }_{3} \stackrel{\omega}{R}_{2}(x)$, then as $v \rightarrow \infty$, it leads to

$$
\stackrel{\omega}{B}\left(\begin{array}{c}
a, b ; c ; v \\
u, \mu
\end{array}\right) \sim v^{-a} \times B(\mu, u) \times{ }_{3} R_{2}\left(\begin{array}{c}
u, a: b ; \frac{1}{v} \\
1-\mu: c
\end{array}\right) .
$$

RECURRENCE RELATIONS. The following recurrence relations for $\stackrel{\omega}{B}$ can be easily derived from its definition and the recurrence relations of $\stackrel{\omega}{R}$ given in [12, equations (8)-(12)].

THEOREM 2.4. The following relations hold:

$$
\begin{aligned}
& (b-a \omega) \stackrel{\omega}{B}\left(\begin{array}{c}
a, b ; c ; v \\
u, \mu
\end{array}\right)=b \stackrel{\omega}{B}\left(\begin{array}{c}
a, b+1 ; c ; v \\
u, \mu
\end{array}\right)-a v \omega \stackrel{\omega}{\omega}\left(\begin{array}{c}
a+1, b ; c ; v \\
u, \mu
\end{array}\right), \\
& (c-a \omega-1) \stackrel{\omega}{B}\left(\begin{array}{c}
a, b ; c ; v \\
u, \mu
\end{array}\right)=(c-1) \stackrel{\omega}{B}\left(\begin{array}{c}
a, b ; c-1 ; v \\
u, \mu
\end{array}\right)-a v \omega \stackrel{\omega}{\omega}\left(\begin{array}{c}
a+1, b ; c ; v \\
u, \mu
\end{array}\right), \\
& (c-b-1) \stackrel{\omega}{B}\left(\begin{array}{c}
a, b ; c ; v \\
u, \mu
\end{array}\right)=(c-1) \stackrel{\omega}{B}\left(\begin{array}{c}
a, b ; c-1 ; v \\
u, \mu
\end{array}\right)-b \stackrel{\omega}{B}\left(\begin{array}{c}
a, b+1 ; c ; v \\
u, \mu
\end{array}\right) \text {, } \\
& \stackrel{\omega}{B}\left(\begin{array}{c}
a, b ; c ; v \\
u, \mu
\end{array}\right)=(c-b) \stackrel{\omega}{B}\left(\begin{array}{c}
a, b ; c+1 ; v \\
u, \mu
\end{array}\right)+b \stackrel{\omega}{B}\left(\begin{array}{c}
a, b+1 ; c+1 ; v \\
u, \mu
\end{array}\right) .
\end{aligned}
$$

Moreover, using [12, equation (17)] and integration by parts to the integral representation of $\stackrel{\omega}{B}$ we obtain the following result.

LEMMA 2.5. With the preceding assumptions, one can easily derive

$$
\stackrel{\omega}{B}\left(\begin{array}{c}
a, b ; c ; v \\
u, \mu
\end{array}\right)=\frac{u+\mu}{u} \stackrel{\omega}{B}\left(\begin{array}{c}
a, b ; c ; v \\
u+1, \mu
\end{array}\right)+\frac{\widehat{L}}{v u} \stackrel{\omega}{B}\left(\begin{array}{c}
a+1, b+\omega ; c+\omega ; v \\
u+1, \mu-1
\end{array}\right)
$$

with

$$
\widehat{L}=\frac{a \Gamma(c) \Gamma(b+\omega)}{\Gamma(b) \Gamma(c+\omega)}
$$

We conclude this section by giving the partial derivatives of the generalized beta function.

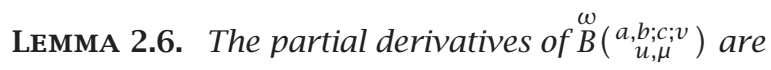

$$
\begin{gathered}
\frac{\partial^{n}}{\partial u^{n}} \stackrel{\omega}{B}\left(\begin{array}{c}
a, b ; c ; v \\
u, \mu
\end{array}\right)=v^{-a} \int_{0}^{\infty}(-1)^{n+1} t^{-1}[\ln (1+t)]^{n}(1+t)^{-\mu-u}{ }_{2} R_{1}\left(a, b ; c ;-\frac{t}{v}\right) d t \\
\frac{\partial^{n}}{\partial v^{n}} \stackrel{\omega}{B}\left(\begin{array}{c}
a, b ; c ; v \\
u, \mu
\end{array}\right)=(-1)^{n}(a)_{n} \stackrel{\omega}{B}\left(\begin{array}{c}
a+n, b ; c ; v \\
u, \mu
\end{array}\right) .
\end{gathered}
$$


Proof. The first formula is obtained by observing that

$$
\frac{\partial^{n}}{\partial u^{n}}\left[t^{u-1}(1+t)^{-\mu-u}\right]=(-1)^{n+1} t^{-1}[\ln (1+t)]^{n}(1+t)^{-\mu-u} .
$$

The second formula is obtained by using the integral representation of ${ }_{2} R_{1}$, that is,

$$
{ }_{2}^{\omega} R_{1}\left(a, b ; c ;-\frac{t}{v}\right)=\widetilde{L} \int_{0}^{1} s^{b / \omega-1}\left(1-s^{1 / \omega}\right)^{c-b-1}\left(1+\frac{t}{v} s\right)^{-a} d s, \quad \widetilde{L}=\frac{1}{\omega \times B(b, c-b)}
$$

and recalling that,

$$
\frac{\partial^{n}}{\partial v^{n}}(v+s t)^{-a}=(-1)^{n}(a)_{n}(v+s t)^{-a-n}
$$

This ends the proof.

3. The generalized incomplete beta functions. We define for $x, \omega>0, \operatorname{Re} u, \operatorname{Re}(a+$ $\mu), \operatorname{Re}(b+\mu)>0$, and $|\arg v|<\pi$, the related functions:

$$
\stackrel{\omega}{B}_{0}^{x}\left(\begin{array}{c}
a, b ; c ; v \\
u, \mu
\end{array}\right)=v^{-a} \int_{0}^{x} t^{u-1}(1+t)^{-\mu-u}{ }_{2}^{\omega} R_{1}\left(a, b ; c ;-\frac{t}{v}\right) d t,
$$

and call it the generalized incomplete beta function, and its companion function,

$$
\stackrel{\omega}{B}_{x}^{\infty}\left(\begin{array}{c}
a, b ; c ; v \\
u, \mu
\end{array}\right)=v^{-a} \int_{x}^{\infty} t^{u-1}(1+t)^{-\mu-u}{ }_{2} \stackrel{\omega}{R}_{1}\left(a, b ; c ;-\frac{t}{v}\right) d t
$$

which may be called the generalized complementary incomplete beta function. In other words, we have

$$
\stackrel{\omega}{B}\left(\begin{array}{c}
a, b ; c ; v \\
u, \mu
\end{array}\right)=\stackrel{\omega}{B}_{0}^{x}\left(\begin{array}{c}
a, b ; c ; v \\
u, \mu
\end{array}\right)+\stackrel{\omega}{B}_{x}^{\infty}\left(\begin{array}{c}
a, b ; c ; v \\
u, \mu
\end{array}\right)
$$

The next theorem lists some differential properties and recurrence relations of these incomplete functions. For simplicity, we let

$$
B_{0}^{x} \triangleq \stackrel{\omega}{B}_{0}^{x}\left(\begin{array}{c}
a, b ; c ; v \\
u, \mu
\end{array}\right), \quad B_{x}^{\infty} \triangleq \stackrel{\omega}{B}_{x}^{\infty}\left(\begin{array}{c}
a, b ; c ; v \\
u, \mu
\end{array}\right) .
$$

THEOREM 3.1. Continue with the preceding notations, to obtain the following formulas:

$$
\begin{aligned}
\stackrel{\omega}{B}_{0}^{x}\left(\begin{array}{c}
a, b ; c ; v \\
u, \mu
\end{array}\right)= & \frac{u+\mu}{u} \stackrel{\omega}{B}_{0}^{x}\left(\begin{array}{c}
a, b ; c ; v \\
u+1, \mu
\end{array}\right)+\frac{x^{u-1}(1+x)^{-\mu-u}}{v^{a}}{ }_{2} R_{1}\left(a, b ; c ;-\frac{x}{v}\right) \\
& +\frac{\hat{L}}{v u} B_{0}^{\omega}\left(\begin{array}{c}
a+1, b+\omega ; c+\omega ; v \\
u+1, \mu-1
\end{array}\right)
\end{aligned}
$$


with $\widehat{L}=a \Gamma(c) \Gamma(b+\omega) / \Gamma(b) \Gamma(c+\omega)$.

$$
\begin{aligned}
\stackrel{\omega}{B}_{x}^{\infty}\left(\begin{array}{c}
a, b ; c ; v \\
u, \mu
\end{array}\right)= & \frac{u+\mu}{u} B_{x}^{\omega}\left(\begin{array}{c}
a, b ; c ; v \\
u+1, \mu
\end{array}\right)-\frac{x^{u-1}(1+x)^{-\mu-u}}{v^{a}}{ }_{2}^{\omega} R_{1}\left(a, b ; c ;-\frac{x}{v}\right) \\
& +\frac{\widehat{L}}{v u} \stackrel{\omega}{B}_{x}^{\infty}\left(\begin{array}{c}
a+1, b+\omega ; c+\omega ; v \\
u+1, \mu-1
\end{array}\right), \\
\frac{d}{d x}\left[x^{1-u} B_{0}^{x}\right] & =(1-u) x^{-u} B_{0}^{x}+v^{-a}(1+x)^{-\mu-u}{ }_{2} R_{1}\left(a, b ; c ;-\frac{x}{v}\right), \\
\frac{d}{d x}\left[(1+x)^{\mu+u} B_{0}^{x}\right] & =(u+\mu)(1+x)^{\mu+u-1} B_{0}^{x}+v^{-a} x^{u-1}{ }_{2}^{\omega} R_{1}\left(a, b ; c ;-\frac{x}{v}\right), \\
\frac{d}{d x}\left[x^{1-u} B_{x}^{\infty}\right] & =(1-u) x^{-u} B_{x}^{\infty}-v^{-a}(1+x)^{-\mu-u}{ }_{2} R_{1}\left(a, b ; c ;-\frac{x}{v}\right), \\
\frac{d}{d x}\left[(1+x)^{\mu+u} B_{x}^{\infty}\right] & =(u+\mu)(1+x)^{\mu+u-1} B_{x}^{\infty}-v^{-a} x^{u-1}{ }_{2}^{\omega} R_{1}\left(a, b ; c ;-\frac{x}{v}\right) .
\end{aligned}
$$

The proof of the formulas (3.5), (3.6), and (3.7) is straight forward from the respective definitions.

4. The probability density function. In a systematic study of generalized pdf and their statistical properties, special functions have played a significant role $[2,3,6,7$, 10]. Chaudhry and Zubair [5, 4] have used modified Bessel functions to extend the gamma function, and then used them to define some densities. Kalla et al. [7] have used a generalized form of hypergeometric function to study a new pdf Ismail Ali et al. [3] have used $\tau$-confluent hypergeometric function to define and study a generalized inverse Gaussian distribution. Here we study a new probability density involving the function defined by (1.2).

The pdf of a random variable $X$ associated with (1.2) is defined by,

$$
f(x)=\frac{v^{-a} x^{u-1}(1+x)^{-\mu-u}{ }_{2} R_{1}(a, b ; c ;-x / v)}{\stackrel{\omega}{B}\left(\begin{array}{c}
a, b ; c ; v \\
u, \mu
\end{array}\right)} \times 1[x>0] .
$$

It is obvious that $\int_{0}^{\infty} f(t) d t=1$. We observe that the behavior of $f(x)$ at zero depends on $u$, that is,

$$
f(0)= \begin{cases}0, & u>1 \\
{\left[v^{a} \stackrel{\omega}{B}\left(\begin{array}{c}
a, b ; c ; v \\
1, \mu
\end{array}\right)\right]^{-1},} & u=1\end{cases}
$$

Moreover, we have $\lim _{x \rightarrow 0^{+}} f(x)=\infty, u<1$, and $\lim _{x \rightarrow \infty} f(x)=0$. It can be easily shown that

$$
\frac{d}{d x} f(x)=\left[\frac{u-1}{x}-\frac{\mu+u}{1+x}-\frac{L}{v} \frac{\stackrel{\omega}{R_{1}}(a+1, b+\omega ; c+\omega ;-x / v)}{{ }_{2}^{\omega} R_{1}(a, b ; c ;-x / v)}\right] f(x),
$$

where $L=a \Gamma(c) \Gamma(b+\omega) / \Gamma(b) \Gamma(c+\omega)$. 


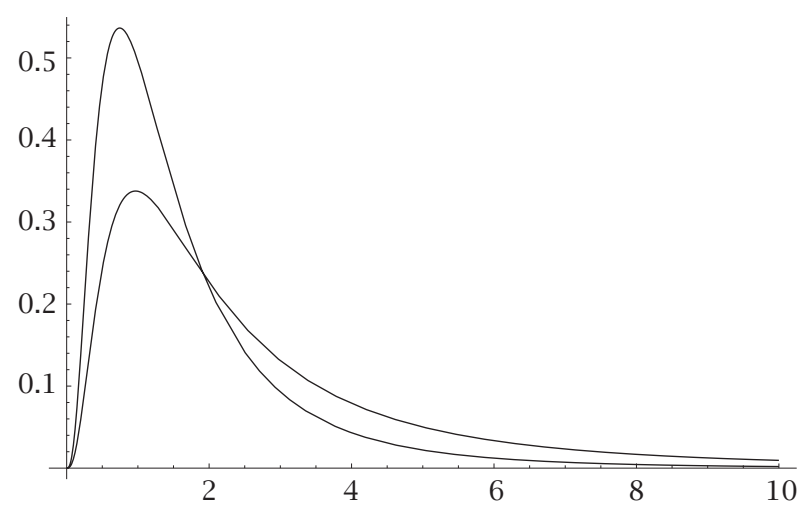

FIGURE 4.1. The probability density function $f(x)$ when $v=1, u=4, m=2$, $a=3, b=2, c=7$. The lower graph represents $f(x)$ when $\omega=4$ whereas the upper graph represents $f(x)$ when $\omega=1$.

Figure 4.1 represents the pdf for the indicated parameters. It shows the effect of the parameter $\omega$.

SPECIAL CASES. If we set $\omega=1$, the density function becomes

$$
f(x)=\frac{v^{-a} x^{u-1}(1+x)^{-\mu-u_{2} F_{1}(a, b ; c ;-x / v)}}{B\left(\begin{array}{c}
a, b ; c ; v \\
u, \mu
\end{array}\right)} \times 1[x>0] .
$$

Furthermore, if we let $b=c$, the density function (4.4) reduces to

$$
f(x)=\frac{x^{u-1}(1+x)^{-\mu-u}(1+x / v)^{-a} \times 1[x>0]}{B(u, \mu+a)_{2} F_{1}(u, a ; u+a+\mu ; 1-1 / v)} .
$$

The beta density function of second kind is recovered from (4.1) when $a=0$

$$
f(x)=\frac{x^{u-1}(1+x)^{-\mu-u}}{B(u, \mu)} \times 1[x>0] .
$$

5. Some statistical function. The aim of this section is to obtain some basic functions associated with the pdf $f(x)$, such as the population moments, the cumulative distribution function (cdf), the survivor function, the hazard rate function, and the mean residue life function.

5.1. Population moments. We derive several types of moments such as the $k$ th moment and the moment generating function. We begin by evaluating the $k$ th moment, since it will be used to obtain the remaining basic moments, such as the mean, the variance and the moment generating function

The $k$ TH Moment. The $k$ th moment about the origin of the random variable $X$ whose pdf $f(x)$ given by (4.1), is defined by

$$
E\left[X^{k}\right] \triangleq \int_{0}^{\infty} t^{k} f(t) d t
$$


By virtue of (4.1), we get

$$
E\left[X^{k}\right]=\frac{v^{-a} \int_{0}^{\infty} t^{k+u-1}(1+t)^{-\mu-u}{ }_{2} R_{1}(a, b ; c ;-t / v) d t}{\stackrel{\omega}{B}\left(\begin{array}{c}
a, b ; c ; v \\
u, \mu
\end{array}\right)}=\frac{\stackrel{\omega}{B}\left(\begin{array}{c}
a, b ; c ; v \\
u+k, \mu-k
\end{array}\right)}{\stackrel{\omega}{B}\left(\begin{array}{c}
a, b ; c ; v \\
u, \mu
\end{array}\right)} .
$$

Now since the mean, expected value of the random variable $X$, is a special case of this moment, namely, the mean is the first moment

$$
E[X] \triangleq \int_{0}^{\infty} t f(t) d t=\frac{\stackrel{\omega}{B}\left(\begin{array}{c}
a, b ; c ; v \\
u+1, \mu-1
\end{array}\right)}{\stackrel{\omega}{B}\left(\begin{array}{c}
a, b ; c ; v \\
u, \mu
\end{array}\right)}
$$

Similarly, we can obtain the variance of the random variable $X, \sigma_{X}^{2}$, using (5.2) with $k=2$, and (5.3), since it is defined as

$$
\sigma_{X}^{2} \triangleq E\left[X^{2}\right]-(E[X])^{2}
$$

Moment GENERATING FUnCtion. The moment generating function of the random variable $X$ is defined by

$$
M(t) \triangleq E\left[e^{t X}\right]=\int_{0}^{\infty} e^{t x} f(x) d x .
$$

To avoid the difficulty of this integration, we observe, using Taylor expansion, that

$$
E\left[e^{t X}\right]=\sum_{k=0}^{\infty} \frac{t^{k}}{k !} E\left[X^{k}\right] .
$$

Using this beside (5.2), we obtain

$$
M(t)=\sum_{k=0}^{\infty} \frac{\stackrel{\omega}{B}\left(\begin{array}{c}
a, b ; c ; v \\
u+k, \mu-k
\end{array}\right)}{\stackrel{\omega}{B}\left(\begin{array}{c}
a, b ; c ; v \\
u, \mu
\end{array}\right)} \times \frac{t^{k}}{k !}
$$

5.2. The distribution function. The $\operatorname{cdf} F(x)$ of the random variable $X$ is given by,

$$
F(x) \triangleq P(X \leq x)=\int_{0}^{x} f(t) d t=\frac{\stackrel{\omega}{B}_{0}^{x}\left(\begin{array}{c}
a, b ; c ; v \\
u, \mu
\end{array}\right)}{\stackrel{\omega}{B}\left(\begin{array}{c}
a, b ; c ; v \\
u, \mu
\end{array}\right)},
$$


hence the survivor function $S(x)$ can be expressed as

$$
S(x) \triangleq P(X \geq x)=1-F(x)=\int_{x}^{\infty} f(t) d t=\frac{\stackrel{\omega}{B}_{x}^{\infty}\left(\begin{array}{c}
a, b ; c ; v \\
u, \mu
\end{array}\right)}{\stackrel{\omega}{B}\left(\begin{array}{c}
a, b ; c ; v \\
u, \mu
\end{array}\right)} .
$$

5.3. The hazard rate function. For a pdf $f(x)$ the hazard rate function is defined by

$$
h(x) \triangleq \frac{f(x)}{S(x)}
$$

Using (5.2) and (5.9), it follows that

$$
h(x)=\frac{v^{-a} x^{u-1}(1+x)^{-\mu-u}{ }_{2} R_{1}(a, b ; c ;-x / v)}{\stackrel{\omega}{B}_{x}^{\infty}\left(\begin{array}{c}
a, b ; c ; v \\
u, \mu
\end{array}\right)} \times 1[x>0] .
$$

A particular case of the hazard function $h(x)$ results when $\omega=1$, that is,

$$
h(x)=\frac{v^{-a} x^{u-1}(1+x)^{-\mu-u_{2} F_{1}(a, b ; c ;-x / v)}}{B_{x}^{\infty}\left(\begin{array}{c}
a, b ; c ; v \\
u, \mu
\end{array}\right)} \times 1[x>0] .
$$

Further, for $b=c$, the hazard function (5.12) reduces to

$$
h(x)=\frac{x^{u-1}(1+x)^{-\mu-u}(v+x)^{-a} \times 1[x>0]}{B_{x}^{\infty}\left(\begin{array}{c}
a, b ; b ; v \\
u, \mu
\end{array}\right)} .
$$

The hazard function of the beta distribution of the second kind is recovered from (5.11) when $a=0$

$$
h(x)=\frac{x^{u-1}(1+x)^{-\mu-u}}{B_{x}^{\infty}(u, \mu)} \times 1[x>0] .
$$

5.4. The mean residue life function. For a random variable $X$, the mean residue life function is defined by

$$
K(x)=E[X-x / X \geq x]=\frac{\int_{x}^{\infty}(t-x) f(t) d t}{S(x)}=\frac{\int_{x}^{\infty} t f(t) d t}{S(x)}-x .
$$

Now since

$$
\int_{x}^{\infty} t f(t) d t=\frac{\stackrel{\omega}{B}_{x}^{\infty}\left(\begin{array}{c}
a, b ; c ; v \\
u+1, \mu-1
\end{array}\right)}{\stackrel{\omega}{B}\left(\begin{array}{c}
a, b ; c ; v \\
u, \mu
\end{array}\right)}
$$




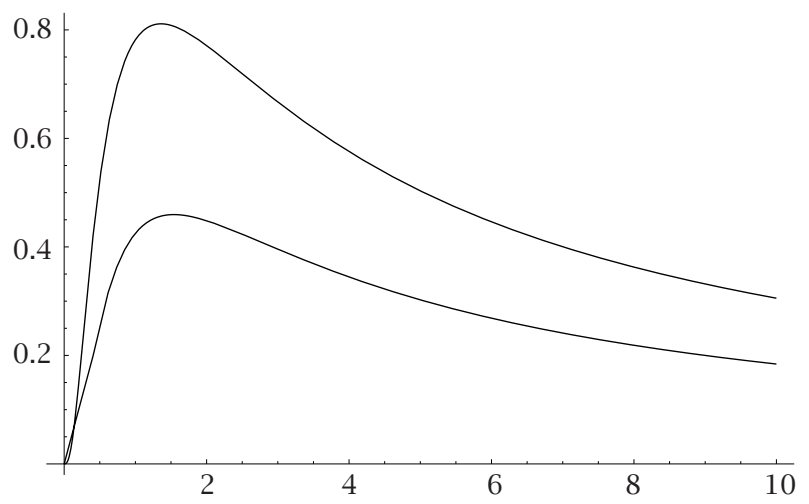

FIGURE 5.1. The hazard function $h(x)$ when $v=1, u=4, m=2, a=3$, $b=2, c=7$. The lower graph represents $h(x)$ when $\omega=4$ whereas the upper graph represents $h(x)$ when $\omega=1$.

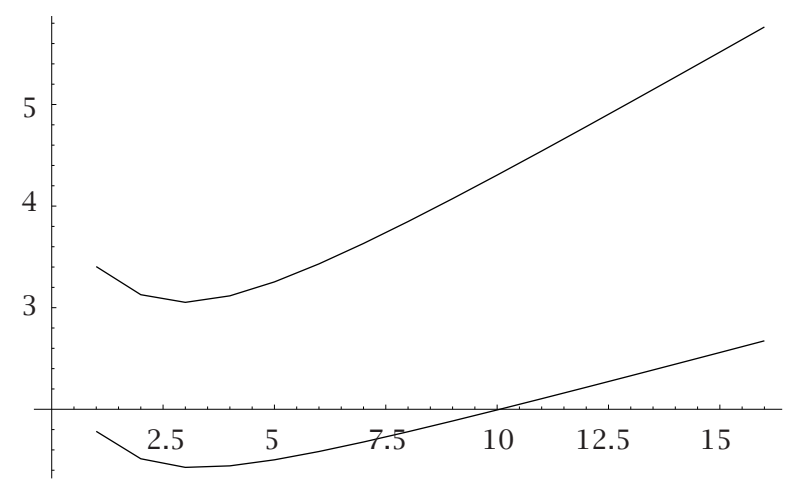

FIGURE 5.2. The mean residue life function $K(x)$ when $v=1, u=4, m=2$, $a=3, b=2, c=7$. The lower graph represents $K(x)$ when $\omega=1$ whereas the upper graph represents $K(x)$ when $\omega=4$.

therefore, using this and (5.9), we get

$$
K(x)=\frac{\stackrel{\omega}{B}_{x}^{\infty}\left(\begin{array}{c}
a, b ; c ; v \\
u+1, \mu-1
\end{array}\right)}{\stackrel{\omega}{B}_{x}^{\infty}\left(\begin{array}{c}
a, b ; c ; v \\
u, \mu
\end{array}\right)}-x .
$$

For $a=0$, we obtain the mean residue life function $K(x)$ of the beta distribution of the second kind

$$
K(x)=\frac{B_{x}^{\infty}(u+1, \mu-1)}{B_{x}^{\infty}(u, \mu)}-x
$$


Figures 5.1 and 5.2 represent the hazard function $h(x)$ and the mean residue life function $K(x)$. They show the effect of the parameter $\omega$.

\section{REFERENCES}

[1] A. Abramowitz and I. Stegun, Handbook of Mathematical Functions, Dover Publications, New York, 1972.

[2] A. Al-Zamel, On a generalized gamma-type distribution with $\tau$-confluent hypergeometric function, Kuwait J. Sci. Engrg. 28 (2001), 25-36.

[3] I. Ali, S. L. Kalla, and H. G. Khajah, A generalized inverse Gaussian distribution with T-confluent hypergeometric function, Integral Transform. Spec. Funct. 12 (2001), no. 2, 101-114.

[4] M. A. Chaudhry and S. M. Zubair, Generalized incomplete gamma functions with applications, J. Comput. Appl. Math. 55 (1994), 99-124.

[5] __ On an extension of generalized incomplete gamma functions with applications, J. Austral. Math. Soc. Ser. B 37 (1996), 392-405.

[6] S. L. Kalla and B. N. Al-Saqabi, Further results on a unified form of gamma-type distributions, Fract. Calc. Appl. Anal. 4 (2001), no. 1, 91-100.

[7] S. L. Kalla, B. N. Al-Saqabi, and H. G. Khajah, A unified form of gamma-type distributions, Appl. Math. Comput. 118 (2001), no. 2-3, 175-187.

[8] K. Kobayashi, On generalized gamma functions occurring in diffraction theory, J. Phys. Soc. Japan 60 (1991), no. 5, 1501-1512.

[9] W. Magnus, F. Oberhettinger, and R. P. Soni, Formulas and Theorems for the Special Functions of Mathematical Physics, 3rd ed., Springer-Verlag, New York, 1966.

[10] A. M. Mathai, A Handbook of Generalized Special Functions for Statistical and Physical Sciences, The Clarendon Press Oxford University Press, New York, 1993.

[11] A. P. Prudnikov, Y. A. Brychkov, and O. I. Marichev, Integrals and Series. Vol. 3. Direct Laplace Transforms, Gordon and Breach Science Publishers, New York, 1992.

[12] N. Virchenko, S. L. Kalla, and A. Al-Zamel, Some results on a generalized hypergeometric function, Integral Transform. Spec. Funct. 12 (2001), no. 1, 89-100.

[13] N. A. Virchenko, On some generalizations of the functions of hypergeometric type, Fract. Calc. Appl. Anal. 2 (1999), no. 3, 233-244.

Y. BEN NAKHI: KUWAit UnIVERSITY, DePARTMENT OF MATHEMATICS AND COMPUTER SCIENCE, P.O. BOX 5969, SAFAT 13060, KUWAIT

E-mail address: jasi@sci.kuniv. edu.kw

S. L. Kalla: Kuwait University, Department of Mathematics and Computer SCience, P.O. BOX 5969, SAFAT 13060, KUWAIT

E-mail address: ka11a@sci.kuniv.edu.kw 


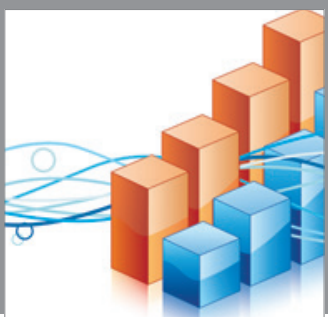

Advances in

Operations Research

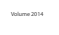

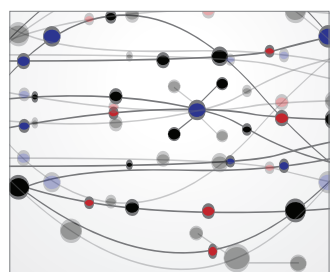

\section{The Scientific} World Journal
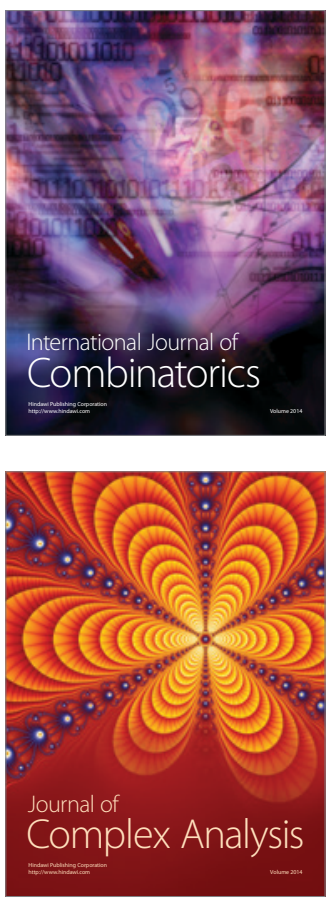

International Journal of

Mathematics and

Mathematical

Sciences
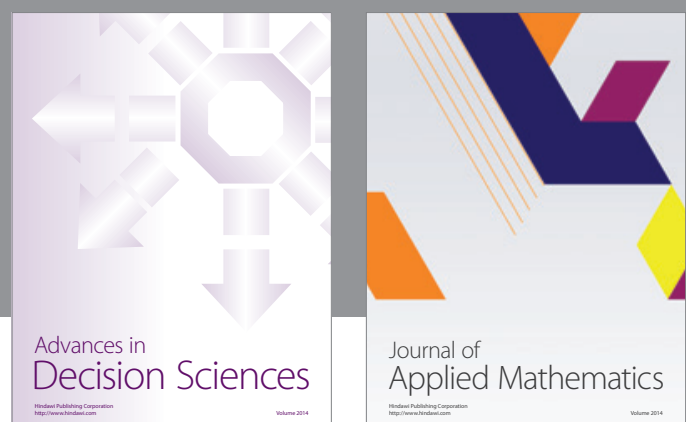

Journal of

Applied Mathematics
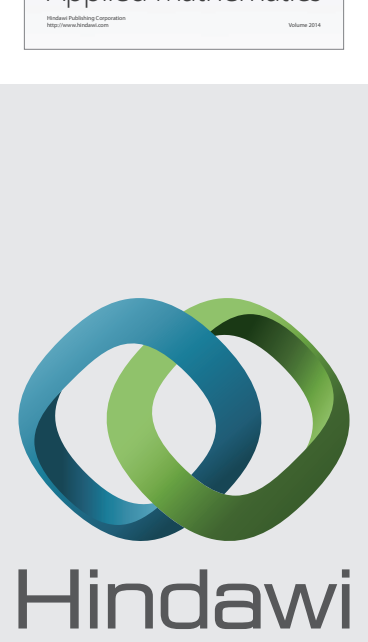

Submit your manuscripts at http://www.hindawi.com
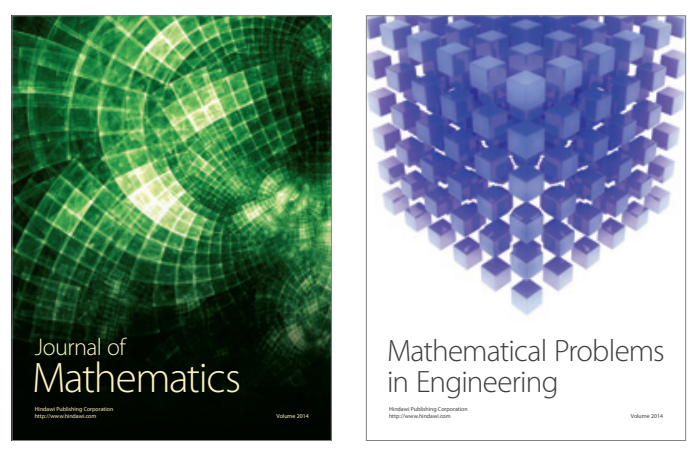

Mathematical Problems in Engineering
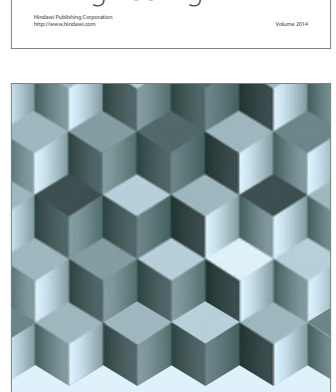

Journal of

Function Spaces
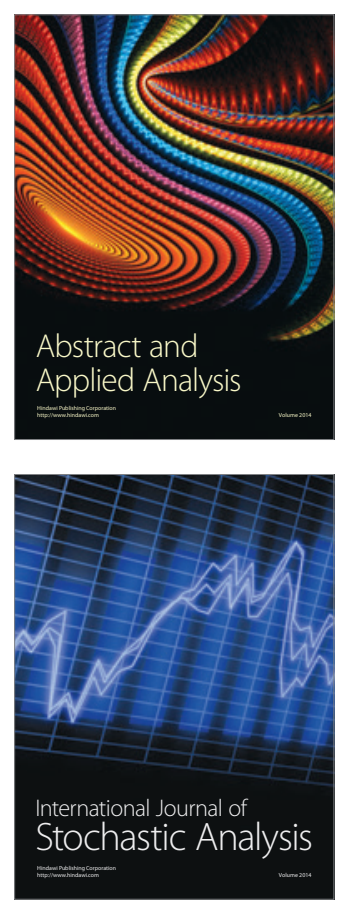

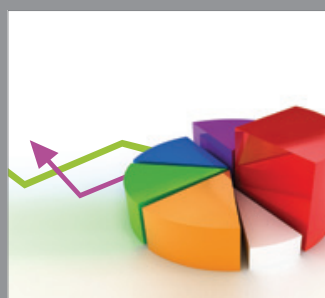

ournal of

Probability and Statistics

Promensencen
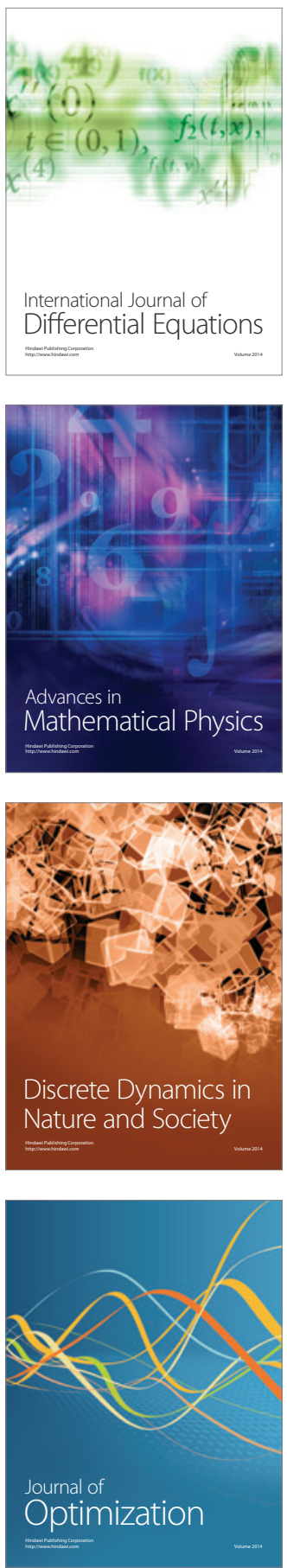